\title{
Ubiquitination by SAG regulates macrophage survival/death and immune response during infection
}

\author{
SC Chang ${ }^{1}$ and JL Ding, ${ }^{*}$
}

The checkpoint between the life and death of macrophages is crucial for the host's frontline immune defense during acute phase infection. However, the mechanism as to how the immune cell equilibrates between apoptosis and immune response is unclear. Using in vitro and ex vivo approaches, we showed that macrophage survival is synchronized by SAG (sensitive to apoptosis gene), which is a key member of the ubiquitin-proteasome system (UPS). When challenged by pathogen-associated molecular patterns (PAMPs), we observed a reciprocal expression profile of pro- and antiapoptotic factors in macrophages. However, SAG knockdown disrupted this balance. Further analysis revealed that ubiquitination of Bax and SARM (sterile $\alpha$ - and HEAT/armadillo-motif-containing protein) by SAG-UPS confers survival advantage to infected macrophages. SAG knockdown caused the accumulation of proapoptotic Bax and SARM, imbalance of Bcl-2/Bax in the mitochondria, induction of cytosolic cytochrome $c$ and activation of caspase-9 and -3 , all of which led to disequilibrium between life and death of macrophages. In contrast, SAG-overexpressing macrophages challenged with PAMPs exhibited upregulation of protumorigenic cytokines (IL-1 $\beta$, IL-6 and TNF- $\alpha$ ), and downregulation of antitumorigenic cytokine (IL-12p40) and anti-inflammatory cytokine (IL-10). This suggests that SAG-dependent UPS is a key switch between immune defense and apoptosis or immune overactivation and tumorigenesis. Altogether, our results indicate that SAG-UPS facilitates a timely and appropriate level of immune response, prompting future development of potential immunomodulators of SAG-UPS.

Cell Death and Differentiation (2014) 21, 1388-1398; doi:10.1038/cdd.2014.54; published online 2 May 2014

In an infection, the pattern recognition receptors (PRRs) of the macrophages recognize pathogen pattern-associated molecular patterns (PAMPs), leading to phagocytosis of the pathogen, release of cytokines and secretion of antimicrobial peptides. When overwhelmed by pathogens, macrophages may undergo apoptosis, which produces microbicidal reactive oxygen species. ${ }^{1}$ Apoptotic death of macrophages is a strategic sacrifice, representing a severe terminal stage of cellular defense against microbial invasion.

The mitochondria has a decisive role in cell death or survival by controlling apoptosis signals via recruitment of pro- and antiapoptosis factors. ${ }^{2}$ Although it is known that many pathogens regulate apoptosis in the host, ${ }^{3}$ the mechanisms underlying how the host immune cell equilibrates its own death and survival to elicit an optimal immune response is poorly understood. This prompted us to investigate how signaling proteins might regulate the checkpoint between apoptosis or immune response. In this regard, we noted several lines of evidence indicating a hitherto undiscovered phenomenon on the control of host cell death or survival versus immune defense. Firstly, it is proposed that the ubiquitin-proteasome system (UPS)-mediated degradation of $\mathrm{Bcl}-2$ family proteins regulates apoptotic cell death. ${ }^{4}$ Secondly, the sensitive to apoptosis gene (SAG), a key component of UPS, is strongly induced during early infection, ${ }^{5}$ suggesting its role in frontline defense. Thirdly, SARM (sterile $\alpha$ - and HEAT/armadillo-motif-containing protein), an evolutionarily conserved mitochondria-associated protein, ${ }^{6,7}$ which downregulates TLR-TRIF signaling, ${ }^{8}$ exerts a strong proapoptotic killing of infection-activated $\mathrm{T}$ cells during the pathogen-clearance phase. ${ }^{9}$ As SAG confers survival to cancer cells, ${ }^{10,11}$ we hypothesize that SAG and SARM have opposing roles in modulating apoptosis and immune response. Thus, we investigated the dynamic expression profiles of SAG and Bcl-2 (antiapoptotic) and Bax and SARM (proapoptotic) in macrophages challenged with bacterial and viral PAMPs.

We demonstrated that SAG responds dynamically to PAMP stimulation. SAG knockdown abrogates ubiquitination and stabilizes the proapoptotic Bax and SARM proteins, leading to their accumulation in the mitochondria and resulting in intrinsic apoptosis. SAG overexpression in macrophages downregulated the antitumorigenic cytokine (IL-12p40) and anti-inflammatory cytokine (IL-10), but upregulated the protumorigenic cytokines (IL-1 $\beta$, IL-6 and TNF- $\alpha$ ), indicating cellular sensitization to SAG activation. Taken together, we propose that the crossroad between macrophage survival/ death and immune response is synchronized to a large extent by SAG-UPS.

Results

SAG is upregulated in macrophages during early infection. Our previous study with Limulus (horseshoe crab), an invertebrate species, which harbors a very powerful innate

\footnotetext{
${ }^{1}$ Department of Biological Sciences, National University of Singapore, 14 Science Drive 4, Singapore 117543, Singapore

*Corresponding author: JL Ding, Department of Biological Sciences, National University of Singapore, 14, Science Drive 4, Singapore 117543, Singapore. Tel: +65 6516 2776; Fax: +65 6779 2486; E-mail: dbsdj|@ nus.edu.sg

Abbreviations: PAMP, pathogen pattern-associated molecular pattern; SAG, sensitive to apoptosis gene; SARM, sterile $\alpha$ - and HEAT/armadillo-motif-containing protein; UPS, ubiquitin-proteasome system

Received 30.9.13; revised 17.3.14; accepted 20.3.14; Edited by RA Knight; published online 02.5.14
} 
immune system, showed that SAG transcription was induced as early as $3 \mathrm{~h}$ after $P$. aeruginosa infection. ${ }^{5}$ Since heatinactivated $P$. aeruginosa is known to induce immune response in mice, ${ }^{12}$ we used it to determine whether mammalian SAG is triggered in a pseudomonas infection. Macrophage $\mathrm{J} 774$ cells challenged with $1 \times 10^{6} \mathrm{CFU} / \mathrm{ml}$ of heat-inactivated $P$. aeruginosa showed a 10-fold rise in both the SAG mRNA within $4 \mathrm{~h}$ (Figure 1a) and SAG protein level at $12 \mathrm{~h}$ (Figure 1b). Since $P$. aeruginosa LPS was sufficient to trigger an inflammatory response, we further studied its effect on SAG expression in a dose-responsive manner in macrophages. SAG protein was upregulated by $5-9$-fold at $12 \mathrm{~h}$ (Figure $1 \mathrm{~b}$ and Supplementary Figure S1A). The profiles of SAG mRNA and protein expression were reproducible with Escherichia coli LPS (Supplementary Figure S1B).

To further understand how SAG is involved in the signaling pathway(s), we tested its expression profile under stimulation by (i) poly I:C, a dsRNA analog, which triggers the TRIF pathway and (ii) R848, an ssRNA analog, which signals via MyD88. We found that poly I:C $(10 \mu \mathrm{g} / \mathrm{ml})$ induced SAG mRNA and protein by 20 - and 14-fold, respectively, at 4 and $12 \mathrm{~h}$, whereas R848 (25 ng/ml) showed minimal effect throughout the challenge (Figures $1 \mathrm{c}$ and d). As SAG activity appears unresponsive to R848, henceforth, we challenged macrophages with LPS and poly I:C.

The early induction of SAG by $P$. aeruginosa, LPS and poly I:C prompted us to hypothesize that SAG exerts its antiapoptotic activity at the initial phase of infection, to facilitate host innate immune defense rather than early death of the immune-responsive cell.

SAG-knockdown macrophages undergo intrinsic apoptosis during PAMP challenge. To investigate how SAG mediates death or survival of infected macrophages, SAG-specific RNAi was conducted in $\mathrm{J} 774$ cells, achieving
84\% knockdown of SAG (Supplementary Figure S2). Cells treated with scrambled siRNA (control) or SAG-specific siRNA were challenged with LPS $(10 \mathrm{ng} / \mathrm{ml})$ or poly I:C $(10 \mu \mathrm{g} / \mathrm{ml})$ over a time course. The cell viability was evaluated using trypan blue dye exclusion and MTT assays. In SAGexpressing control macrophages, a significant reduction of $20 \%$ cell viability was indicated by MTT assay (Figures $2 a$ and $b$ ). This is plausibly important for anti-microbial defense, albeit a mitochondrial respite. In contrast, SAG-knockdown macrophages treated with LPS and poly I:C exhibited significant $(P<0.01)$ decreases in cell viability by $69.1 \%$ and $83.7 \%$, respectively, at $36 \mathrm{~h}$ (Figure $2 \mathrm{~b}$ ). FACS analysis showed that SAG-knockdown cells challenged with LPS or poly I:C started to exhibit early apoptosis within $3 \mathrm{~h}$, compared with control siRNA-treated cells, which only begun to show apoptosis after $12 \mathrm{~h}$ (Figure 2c). Control wild-type J774 cells (not treated with SAG-siRNA or scramble siRNA) challenged with PAMPs showed specific contribution of SAG to apoptosis of macrophages upon infection (Supplementary Figure S3A). SAG-knockdown cells without PAMP challenge showed minimal apoptosis, suggesting that the increased apoptosis of SAG-knockdown cells is specifically induced by the infection condition (Supplementary Figure S3A).

Next, we analyzed the mitochondrial membrane potential $\left(\Delta \Psi_{\mathrm{mt}}\right)$ of infected $\mathrm{J} 774$ cells. The formation of mitochondrial permeability transition pore (MPTP), which indicates the loss of $\Delta \Psi_{\mathrm{mt}}$, was monitored by the mitochondrial membrane potential-driven uptake of fluorescent dye, tetramethylrhodamine ethyl ester (TMRE). SAG-knockdown cells or control siRNA-treated cells were challenged with LPS $(10 \mathrm{ng} / \mathrm{ml})$ or poly l:C $(10 \mu \mathrm{g} / \mathrm{ml})$ over time, followed by TMRE staining and FACS analysis. Supplementary Figure S3B shows a clear separation in fluorescence signal between unstained and TMRE-stained macrophages. Control siRNA-treated cells challenged with PAMPs induced up to $50 \%$ mitochondria- a

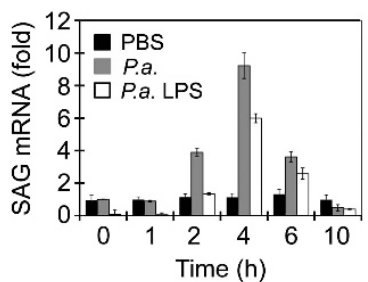

b

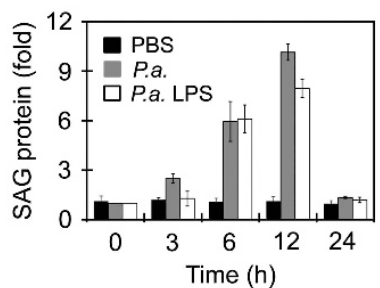

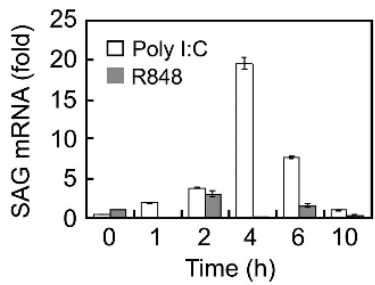

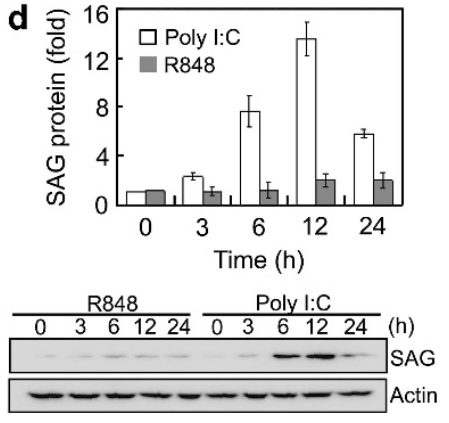

Figure 1 SAG expression is increased in J774 cells at the early stage of challenge with bacterial/viral PAMPs. (a and $\mathbf{b})$ Cells were treated with heat-inactivated P. aeruginosa (P.a., PAO-Iglewski, $1 \times 10^{6} \mathrm{CFU} / \mathrm{ml}$ ), P. aeruginosa LPS (P.a. LPS, $10 \mathrm{ng} / \mathrm{ml}$ ) or phosphate-buffered saline (PBS). (c and d) Cells were treated with viral mimics; polyinosinic-polycytidylic acid (poly l:C) $(10 \mu \mathrm{g} / \mathrm{ml})$ and R848 $(25 \mathrm{ng} / \mathrm{ml})$, representing double-stranded RNA (dsRNA) and single-stranded RNA (ssRNA), respectively. (a and c) mRNA and ( $\mathbf{b}$ and $\mathbf{d}$ ) protein levels of SAG were induced upon treatment by P.a. LPS and poly (I:C), but not by R848. Data are presented as means \pm S.D. ( $n=3$ ) 
a

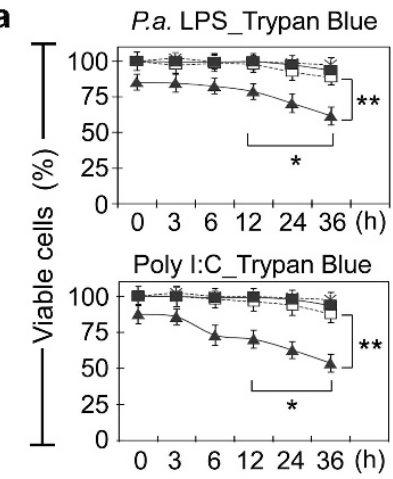

d

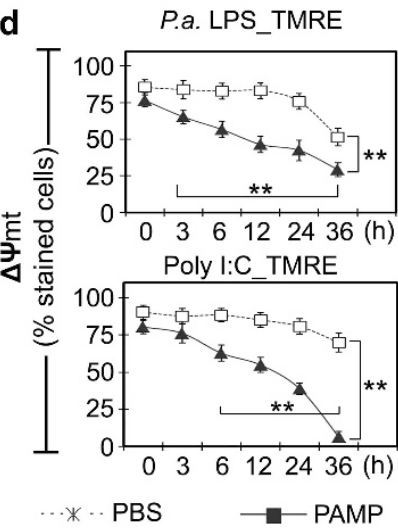

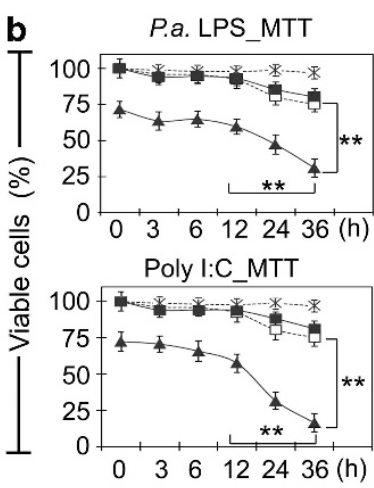

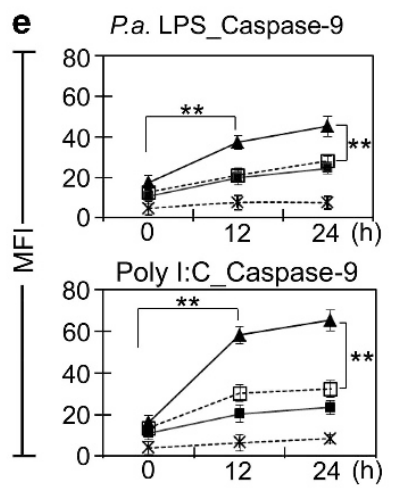

...... Control siRNA+PAMP
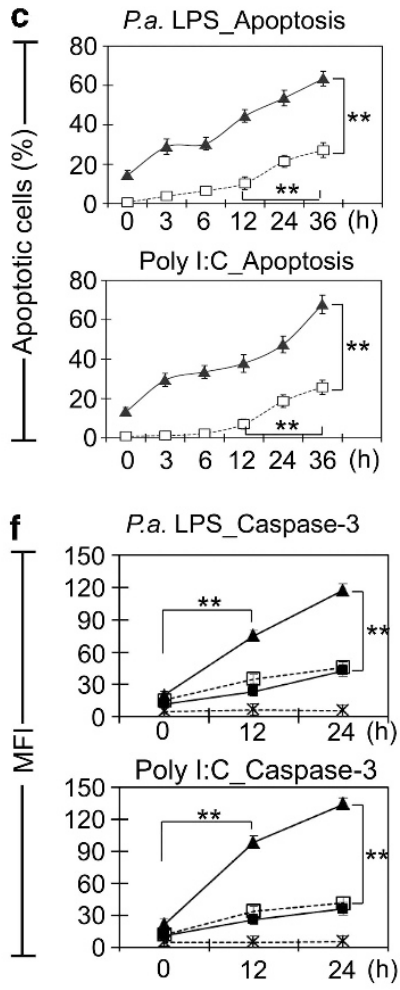

— SAG SIRNA+PAMP

Figure 2 SAG knockdown reduces cell viability and upregulates intrinsic apoptosis in infected macrophages. (a) Trypan blue exclusion test and (b) MTT (3-(4,5-dimethylthiazol-2-yl)-2,5-diphenyltetrazolium bromide) assay demonstrate that knockdown of SAG resulted in loss of cell viability. (c) Annexin V and 7-aminoactinomycin (7-AAD) double staining was conducted to examine the early apoptosis. Representative histograms of apoptosis assay are shown in Supplementary Figure S3A. PAMP-only controls and no-PAMP controls (only SAG small interfering RNA (siRNA) treatment) are shown in Supplementary Figure S3A. (d) TMRE assay show mitochondrial dysfunction when J774 cells were challenged with LPS (10 ng/ml) or polyinosinic-polycytidylic acid (poly l:C) $(10 \mu \mathrm{g} / \mathrm{ml})$. Quantitative results show \% of cells stained with TMRE. No PAMP controls (only SAG siRNA or PBS) and fluorescence-activated cell sorting (FACS) histograms are shown in Supplementary Figure S3B. (e and f) Caspase- 9 and -3 activities in cells exposed to PAMPs for 0,12 and $24 \mathrm{~h}$ were determined based on the hydrolysis of caspase-specific substrate (see Materials and Methods). Representative histograms of caspase assay are shown in Supplementary Figure S3C. Results are expressed as mean fluorescence intensity $(\mathrm{MFI}) \pm$ S.D. $(n=3) .{ }^{*} P<0.05$; ${ }^{* *} P<0.01$

mediated cell death at $36 \mathrm{~h}$. On the contrary, SAG-knockdown cells showed a significant drop in $\Delta \Psi_{\mathrm{mt}}$ at $36 \mathrm{~h}(70 \%$ with LPS treatment and $93 \%$ with poly I:C treatment) $(P<0.01)$ (Figure 2d). Furthermore, SAG knockdown elevated the activation of caspase- 9 and -3 (Figures $2 e$ and f). Representative histograms of FACS analysis are shown in Supplementary Figure S3C. Taken together, both the loss in $\Delta \Psi_{\mathrm{mt}}$ and the activation and cleavage of caspase-9 corroborate the importance of SAG in conferring mitochondriaspecific antiapoptosis to macrophages during early infection.

The ratio of pro- and antiapoptotic factors is regulated by SAG, suggesting a kinetic switch between macrophage death/survival in response to infection. To identify critical regulator(s) of SAG-mediated survival of macrophages during early infection, we examined transcription profiles of selected apoptotic factors, including Bcl-2, Bax, SARM, Noxa and Bcl-xL, based on the literature. ${ }^{4,6,8-10}$ Supplementary Figures S4A and S4B show that neither Noxa (proapoptotic) nor Bcl-xL (antiapoptotic) responded to PAMP challenge, suggesting that these proteins may not directly participate in macrophage survival under the infection conditions studied. Subsequently, we focused on examining the profiles of antiapoptotic Bcl-2 and SAG and proapoptotic Bax and SARM to understand how SAG regulates macrophage survival during infection. Intracellular staining followed by FACS analysis of $\mathrm{J} 774$ cells that had been treated with LPS $(10 \mathrm{ng} / \mathrm{ml})$ or poly l:C $(10 \mu \mathrm{g} / \mathrm{ml})$ showed bimodal expression of the proapoptotic SARM and Bax as early as $3 \mathrm{~h}$ and again at $24 \mathrm{~h}$. In contrast, both the antiapoptotic SAG and $\mathrm{Bcl}-2$ appeared maximally at $12 \mathrm{~h}$ (Figure $3 \mathrm{a}$ (i and iii), left panels). SAG siRNA knockdown abolished these expression profiles (Figure $3 a$ (ii and iv), right panels). Representative histograms of FACS analysis are shown in Supplementary Figure S5. These results are confirmed by immunoblot analysis (Figure $3 b$ ). The rate and timing of expression of the pro- and antiapoptotic proteins appear reciprocal. The balance is in favor of proapoptotic factors reaching a first peak as early as $3 \mathrm{~h}$ of infection and resurging at $24 \mathrm{~h}$, which may explain how the infected cells switch to apoptosis beyond $24 \mathrm{~h}$ PAMP stimulation. SAG-knockdown cells maintained the expression of Bcl-2, but the balance of expression between the pro- and antiapoptosis factors was disrupted; from $3 \mathrm{~h}$ onwards, the expression of the proapoptotic Bax and SARM was sustained at high levels (Figure $3 \mathrm{~b}$, red box) instead of the bimodal peaks observed with control 


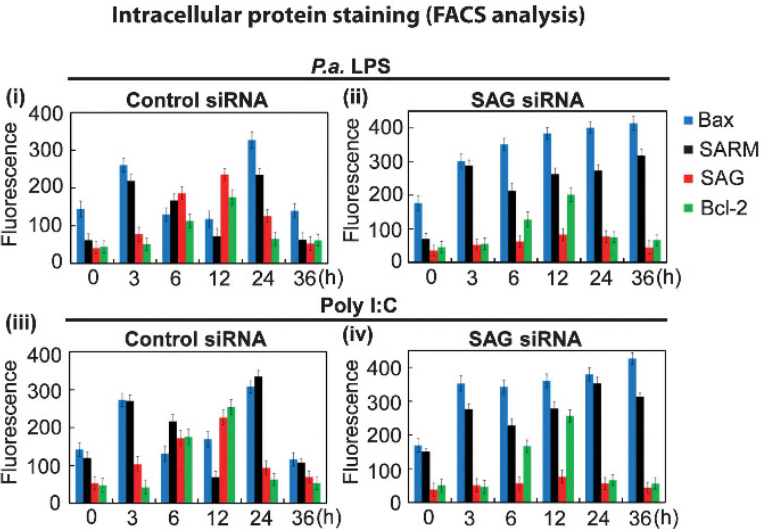

b

Western blotting analysis

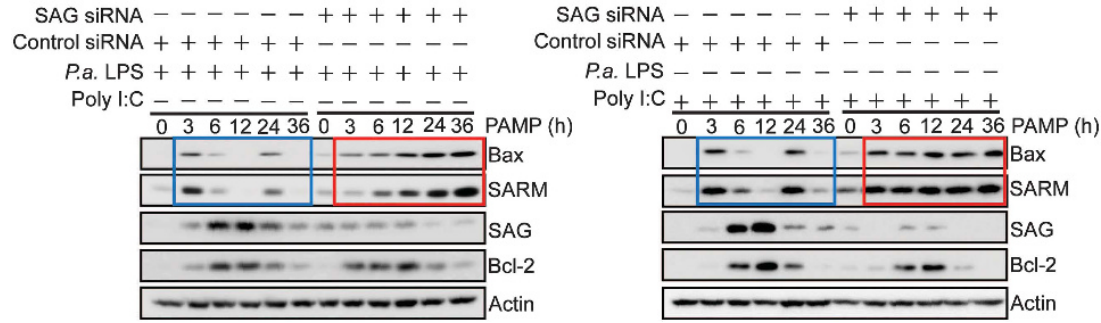

Figure 3 SAG regulates apoptosis of J774 macrophages by modulating the balance between anti- and proapoptotic factors in a timely manner. LPS (10 $\mathrm{ng} / \mathrm{ml}$ ) and polyinosinic-polycytidylic acid (poly l:C) (10 $\mu \mathrm{g} / \mathrm{ml}$ ) stimulation resulted in bimodal peaks (at 3 and $24 \mathrm{~h}$ ) of proapoptotic Bax and SARM, and a single peak (over 6-12 $\mathrm{h}$ ) each of antiapoptotic SAG and Bcl-2 (a (i and iii) and $\mathbf{b}$, blue box). SAG-knockdown cells maintained expression of Bcl-2 but sustained the expression of the proapoptotic Bax and SARM at high levels (over 3-36 h; a (ii and iv) and red box in b). The PAMP-challenged cells were examined by both (a) intracellular staining (see FACS analysis data; Supplementary Figure S5) and (b) immunoblotting analysis. Data are presented as fluorescence intensity \pm S.D. $(n=3)$

SAG-expressing cells (Figure $3 \mathrm{~b}$, blue box). This trend is consistent for both LPS and poly (I:C) treatments, suggesting that SAG may be a dynamic master regulator of macrophage death/survival during infection, without which apoptosis ensues.

While the bimodal profile of Bax and SARM mRNAs was maintained during infection of SAG-knockdown cells (Supplementary Figure S4A), we observed a sustained rise in the Bax and SARM proteins (Figure $3 a$ (ii and iv) and Figure $3 b$, red box), suggesting that the accumulation of Bax and SARM proteins was attributable to a posttranscriptional effect. SAG is known to elicit E3 ubiquitin ligase activity in the ubiquitination of specific target proteins leading to their proteasomal degradation. ${ }^{11}$ Therefore, it is conceivable that the balance of pro- and antiapoptotic factors in macrophages under infection might be regulated via SAG-dependent ubiquitination pathway.

SAG-dependent UPS regulates proapoptotic Bax and SARM. To test whether Bax and/or SARM may be a direct target of the UPS, a proteasome inhibitor, MG132, was administered into $\mathrm{J} 774$ cells. Figure $4 \mathrm{a}$ shows that Bax and SARM were accumulated under proteasome inhibition, indicating the involvement of UPS. In addition, SAG knockdown caused the accumulation of Bax and SARM proteins, suggesting that SAG regulates the UPS-mediated turnover of Bax and SARM. We then examined the degradation rate (half-life) of the proteins after blocking de novo protein synthesis with cycloheximide $(\mathrm{CHX})$ while challenging the cells with LPS $(10 \mathrm{ng} / \mathrm{ml})$ or poly I:C $(10 \mu \mathrm{g} / \mathrm{ml})$. Results showed that SAG knockdown stabilized Bax and SARM proteins by up to $3 \mathrm{~h}$ of $\mathrm{CHX}$ treatment (Figure $4 \mathrm{~b}$ ). A twofold increase in the half-life of Bax and SARM was observed: from $<100 \mathrm{~min}$ in control siRNA-treated cells to $>180 \mathrm{~min}$ in SAG-knockdown cells (Figure 4c). Thus, the absence of the antiapoptotic SAG prolonged the lifespan of Bax and SARM proteins. Plausibly in an infection, SAG facilitates the degradation of proapoptotic Bax and SARM, to sustain cell survival.

If Bax and SARM are direct targets of SAG-dependent UPS, the inhibition of SAG activity should conceivably result in the attenuation of ubiquitinated forms of these proapoptotic proteins. To test this hypothesis, protein extracts of J774 cells, verified to contain Bax, SARM, SAG and Bcl-2 (Figure 4d), were treated with MG132 (or SAG siRNA or control siRNA). Then, the cell lysates were co-immunoprecipitated with Bax or SARM antibody, followed by immunodetection using antiubiquitin antibody. To confirm that observed ubiquitination was due to Bax (or SARM) proteins, the immunoprecipitation (IP) with ubiquitin followed by immunoblotting with Bax (or SARM) was performed. Co-IP shows that both Bax and SARM pulled down SAG (Figure 4e). Inhibition of proteasome increased the levels of ubiquitinated Bax and SARM. Consistently, we observed a decrease in the high-molecular-weight ubiquitinated Bax (HMW Bax-(Ub) $)_{n}$ ) and SARM $\left(\mathrm{HMW}\right.$ SARM- $\left.(\mathrm{Ub})_{n}\right)$ in SAG-silenced cells, compared with control siRNA-treated cells. Furthermore, Co-IP using Bcl-2 as bait showed that $\mathrm{Bcl}-2$ ubiquitination was not regulated by 
a

$132-+-$

SAG SiRNA - - + -

Control siRNA - - - +

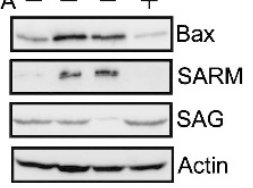

C

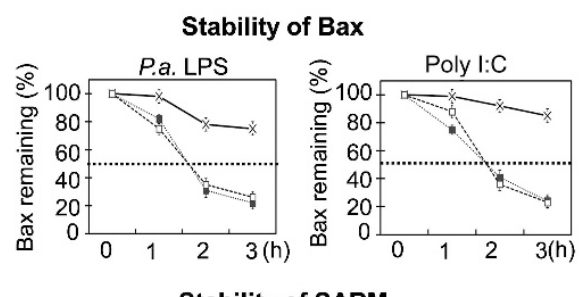

Stability of SARM
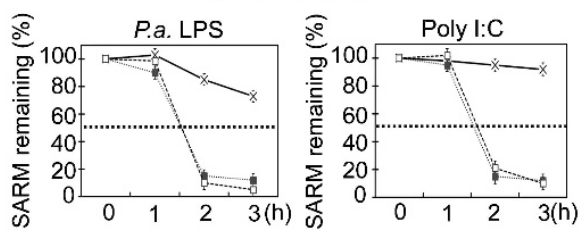

- - SAG SIRNA+ PAMP

.... Control siRNA+ PAMP

$-\square \cdot$ PBS+ PAMP

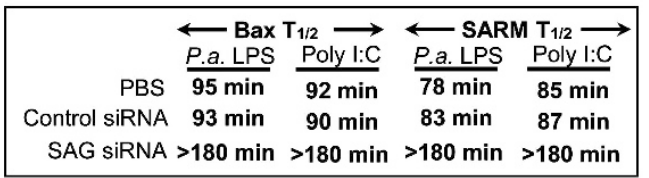

b SAG SiRNA $\ldots \ldots++++\ldots+\ldots$

Control siRNA $----\ldots--++++---$

P.S. LPS ----++++++++++++

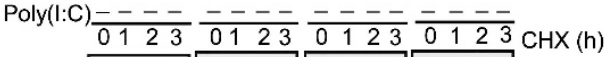

IB: SARM

SAG SIRNA $++++\ldots-\ldots-\ldots$

Control siRNA ----++++----

P.s. LPS - - - - - - - -

Poly(l:C) $++++\frac{++++t++++}{0+12}$

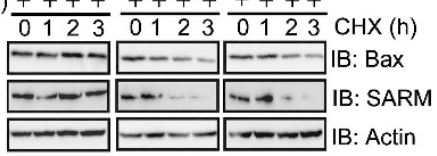

d J774 cell lysate before IP

SAG SIRNA --+---+--

Control siRNA ---+---+

P.S. LPS -++++---

Poly(I:C) -----++++

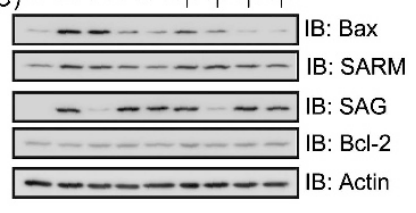

e

MG132 $\frac{\text { IP: Bax }}{-+--+---}$

SAG SIRNA - - + - - + -

Control siRNA - - - + - - +

P.a. LPS -++++---

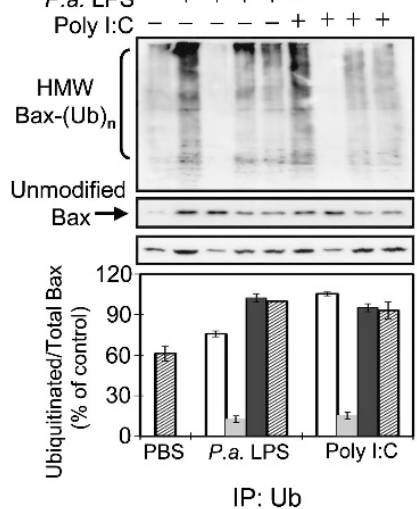

MG132

SAG SiRNA - - + - - + -

Control siRNA - - - + - - +

P.a. LPS -++++---

Poly I:C $-\ldots--++++$

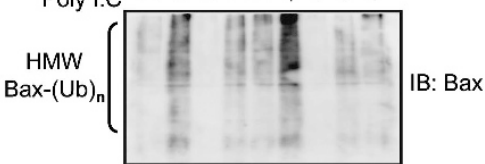

IB: Ub

IB: Bax

IB: SAG

口MG132

SAG SIRNA

- Control siRNA

a NT

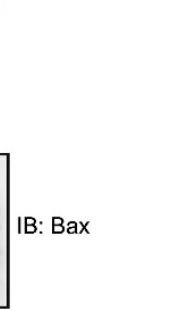

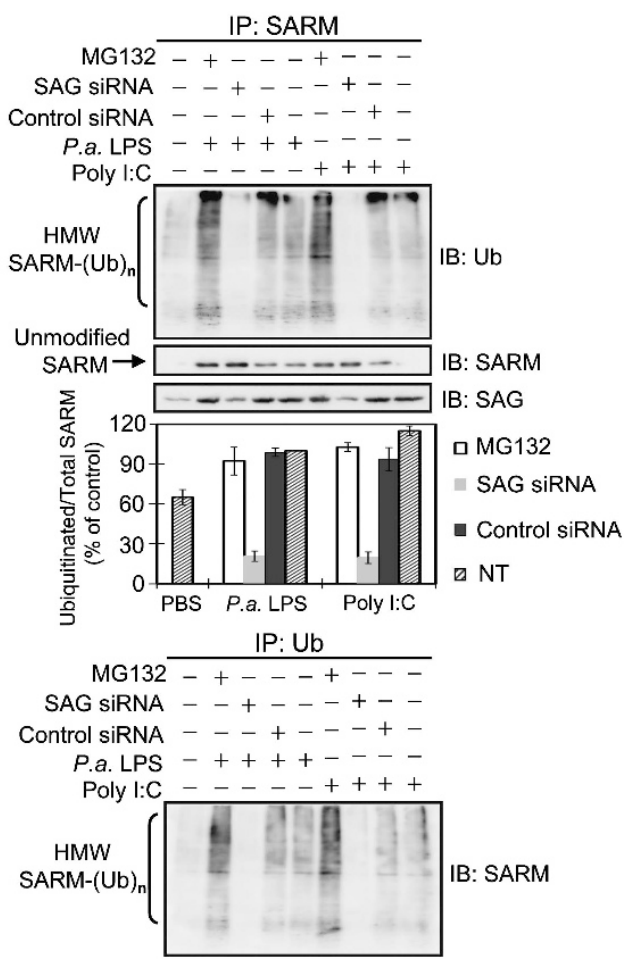


SAG (Supplementary Figure S6). IP with control IgG demonstrated the specificity of the assay (Supplementary Figure S7). These data also corroborate that both Bax and SARM (but not Bcl-2) are specific substrates of SAG-dependent ubiquitination, which is enhanced under infection when the macrophage encounters an apoptosis stimulus. To show that our observations are not restricted to only one cell line, we performed key experiments in another macrophage cell line, RAW264.7. Results show consistent profile of SAG induction, cytochrome $c$ release and ubiquitin activity upon PAMP stimulation (Supplementary Figure S8), suggesting that ubiquitination of apoptosis factors by SAG-UPS indeed regulates macrophage survival/death and its response to infection.

SAG hijacks proapoptotic cytoplasmic Bax and SARM for ubiquitination: observations in primary BMDM. To ensure that our findings in $\mathrm{J} 774$ and RAW cells are physiologically relevant, we performed ex vivo experiments using bone marrow-derived macrophages (BMDM) from BALB/c mice. Consistently, we found that SAG was upregulated in BMDM in response to LPS $(10 \mathrm{ng} / \mathrm{ml})$ and poly I:C $(10 \mu \mathrm{g} / \mathrm{ml})$, but not to R848 (Figure $5 \mathrm{a})$. To further affirm that SAG mediates ubiquitination of Bax and SARM in primary cells, we examined their potential interactions and subcellular colocalization in BMDM challenged with PAMPs. As SAG is cytosolic (Figure $5 b$ ), we fractionated the cell lysate and used the cytosolic extract for Co-IP. Before IP experiments, immunoblotting analysis of whole-cell lysates shows the presence of Bax, SARM, SAG and Bcl-2 in primary BMDM cells (Figure $5 c$ ). Figure $5 d$ shows that under either LPS $(10 \mathrm{ng} / \mathrm{ml})$ or poly I:C $(10 \mu \mathrm{g} / \mathrm{ml})$ challenge, there were interactions between both SAG-Bax and SAG-SARM in the cytosol. It is plausible that SAG binds the cytosolic Bax and SARM and directs these proapoptotic factors toward ubiquitination. Supportive of this speculation was that silencing SAG abrogated the ubiquitination activity, causing the accumulation of unubiquitinated (unmodified) Bax and SARM, but not Bcl-2, in the mitochondria (Figure 5e). To confirm the contribution by SAG in intrinsic apoptosis, we tested the release of cytochrome $c$ by the infected BMDM. We found that $S A G$ knockdown increased the cytosolic cytochrome $c$ at the expense of mitochondrial cytochrome $c$ (Figure 5f). Taken together, we have shown that infection causes SAG to interact with the cytosolic forms of proapoptotic Bax and SARM, hijacking them from the mitochondria and diverting these proteins toward ubiquitination and turnover. This maneuver exerts an influence on the balance of mitochondrial $\mathrm{Bcl}-2 / \mathrm{Bax}$ and subsequently sensitizes the macrophages to infection-induced intrinsic apoptosis.

\section{SAG enhances production of protumorigenic cytokines} in primary BMDM. Upon infection, the key functions of macrophages are to release cytokines and attract secondary defense cells from the blood. Proinflammatory cytokines are key mediators of inflammatory responses. ${ }^{13}$ Figure $6 a$ shows that BMDM cells that overexpress SAG significantly increased $(P<0.01)$ the production of proinflammatory/ protumorigenic cytokines (IL-1 $\beta$, IL- 6 and TNF- $\alpha$ ), whereas $S A G$ knockdown significantly reduced $(P<0.01)$ the production of these cytokines. Concordantly, $S A G$ overexpression downregulated IL-12p40, a proinflammatory/antitumorigenic cytokine. ${ }^{14-16}$ Thus, SAG appears to have a dual role in modulating both tumor growth and immune cell survival. We further measured IL-10, an anti-inflammatory cytokine, known to be regulated by LPS. ${ }^{17-19}$ Figure $6 a$ shows that SAG-knockdown cells challenged with LPS produced high levels $(P<0.01)$ of $\mathrm{IL}-10$, whereas $S A G$ overexpression significantly reduced $(P<0.05)$ the production of IL-10. Thus in infected macrophages, SAG seems to regulate negatively both proinflammatory IL-12p40 and anti-inflammatory IL-10. Therefore, induction of the proinflammatory cytokines (IL-1, IL-6 and TNF- $\alpha$ ) is unlikely attributable to a general increase in the survival advantage resulting from the overexpression of SAG. Rather, there appears to be a tight control of apoptosis in macrophages at early infection, during which SAG-UPS is induced to perform an intricate balance between apoptosis and immune response. Figure $6 \mathrm{~b}$ depicts a model to illustrate the potential role of SAG-UPS linking immune defense and apoptosis or immune overactivation and tumorigenesis.

\section{Discussion}

At the frontline of immune defense, macrophages undergo apoptosis as an optimal strategy against microbial infection. However, the mechanism as to how macrophages equilibrate between apoptosis and immune response is unclear. Here, we show for the first time that under infection, SAG acts as a survival determinant for macrophages by interacting dynamically with the pro- and antiapoptotic factors, which coregulate the life or death of the cells. Coincidently, the SAG promoter harbors multiple transcription factor binding sites, including $\mathrm{AP}-1$ and NF- $\kappa \mathrm{B}$, which are upregulated under TLR activation. ${ }^{20}$ Our findings reveal a novel mechanism by which SAG-UPS balances macrophage life or death.

Figure 4 SAG knockdown blocks ubiquitination and degradation of Bax and SARM, which accumulate in PAMP-treated macrophage J774 cells. (a) Cells were treated with MG132 or SAG siRNA to determine the involvement of SAG-proteasome system in the regulation of proapoptotic Bax and SARM. (b) The stability of Bax and SARM was tested in J774 cells with CHX, a protein synthesis inhibitor, and the lifespan of the proteins was examined by western blotting. (c) The half-lives ( $\left.T_{1 / 2}\right)$ of Bax and SARM were quantified by densitometry analysis. (d) Western blot analysis of whole-cell lysates shows the presence of Bax, SARM, SAG and Bcl-2 in the J774 cells (before IP). Actin was used as a loading control. (e) To investigate the association between SAG and Bax (or SARM) in infected cells, total cell lysates were immunoprecipitated with anti-Bax (or antiSARM) antibody, separated by $12 \%$ and $10 \%$ SDS-PAGE, respectively, immunoblotted and probed with SAG, ubiquitin, Bax or SARM antibodies. SAG-knockdown attenuated the high-molecular-weight (HMW) modified forms of Bax and SARM (but not that of Bcl-2; see Supplementary Figure S6A). Arrows indicate unmodified (un-ubiquitinated) Bax or SARM. A densitometric analysis of ubiquitinated Bax or SARM, relative to total Bax or SARM is plotted (mean \pm S.D., $n=3$ ). To confirm that the observed ubiquitination is due to Bax (or SARM) proteins, IP with ubiquitin, followed by immunoblotting with Bax (or SARM) was performed. IP with control rabbit IgG demonstrates the specificity of the assay (Supplementary Figure S7A). IgG, immunoglobulin G; SDS-PAGE, sodium dodecyl sulfate-polyacrylamide gel electrophoresis 
a
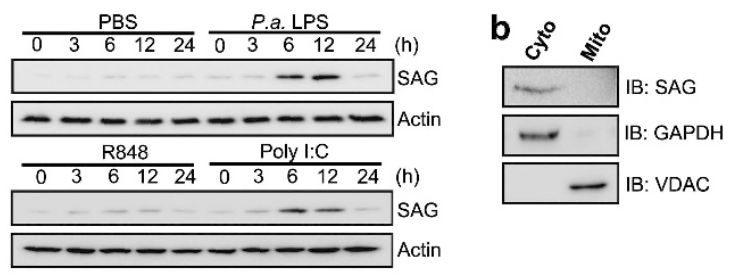

d

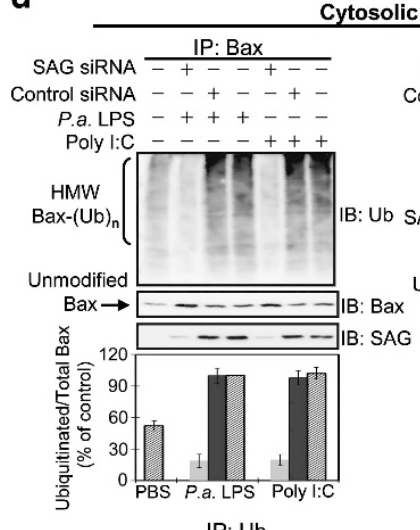
SAG SiRNA $\frac{\text { IP: Ub }}{-+-+--}$
Control siRNA - - + - - +
P.a. LPS -+++---

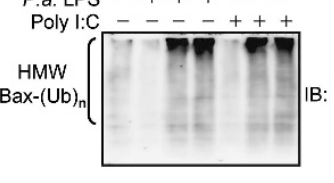

f

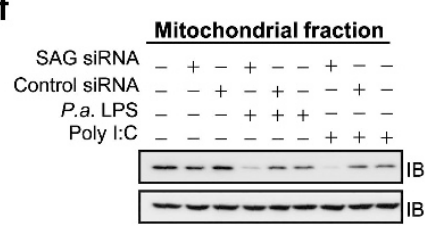

Cytosolic fraction

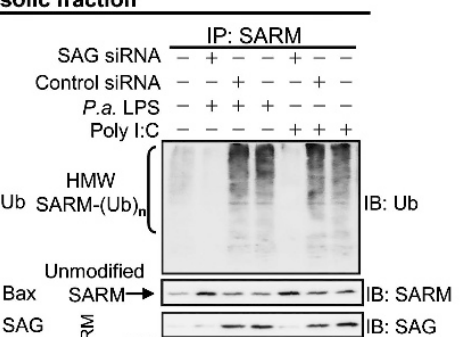

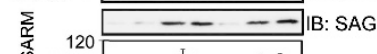

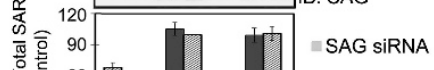

言

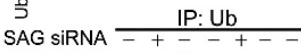

Control SiRNA --+--+

P.a. LPS -+++---

Poly I:C $-\cdots-+++$

HMW

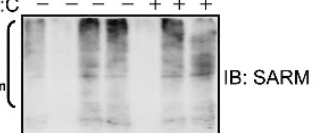

Bax SARM-(Ub)
C BMDM cell lysate before IP

SAG SIRNA -+--+--

Control siRNA --+--+-

P.s. LPS -+++---

Poly(I:C) ----+++

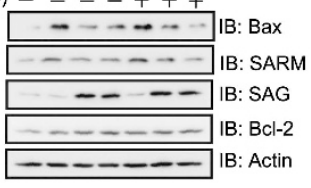

e

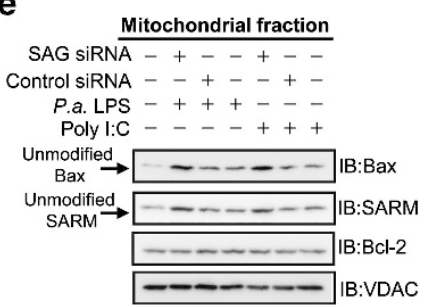
害过

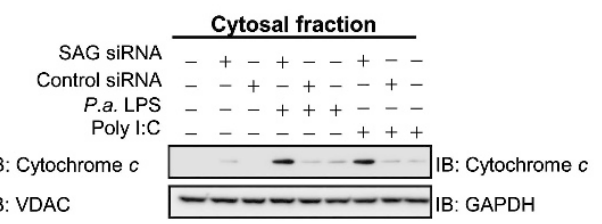

Figure 5 SAG knockdown blocks ubiquitination of Bax and SARM in the cytosol of primary BMDM cells, with increased cytochrome $c$ release. (a) The induction of SAG in infected BMDM was immunodetected. (b) Cells were pretreated with, polyinosinic-polycytidylic acid (poly l:C) $(10 \mu \mathrm{g} / \mathrm{ml})$ for $12 \mathrm{~h}$, followed by cellular fractionation and western blotting. SAG is primarily expressed in the cytosol. (c) Western blot analysis of whole-cell lysates shows the presence of Bax, SARM, SAG and Bcl-2 in primary BMDM cells (before IP). The role of SAG in the ubiquitination of proapoptotic Bax or SARM was determined by Co-IP of subcellular fractions. Cells were treated with SAG siRNA, small interfering RNA (siRNA) (or control siRNA) for $16 \mathrm{~h}$, followed by PAMP stimulation for $24 \mathrm{~h}$. (d) Cytosolic fraction and (e) mitochondrial fraction. Both SAG-Bax and SAG-SARM immunoprecipitates were found in the cytosolic fraction. A densitometric analysis of the ubiquitinated Bax and SARM relative to total Bax and SARM in the cytosol is provided. Data are representative of means \pm S.D. $(n=3)$. To confirm that the observed ubiquitination is due to Bax (or SARM) proteins, the IP with ubiquitin, followed by immunoblotting with Bax (or SARM) was performed. IP with control rabbit IgG shows the specificity of the assay (Supplementary Figure S7B). (f) Cytochrome $c$ release was examined to determine the levels of intrinsic apoptosis, which is increased in SAG-knockdown BMDM.

The pivotal role of SAG to life is underscored by its evolutionary conservation from yeast to human (Supplementary Figure S9). $S A G^{-1-}$ mice are embryonically lethal, further indicating the fundamental significance of $S A G$ to survival. ${ }^{21}$ With its ROS-scavenging antioxidant activity and E3 ubiquitin ligase activity, SAG was reported to exert antiapoptotic activity and stimulate cancer growth. ${ }^{10,22}$ In agreement, our data demonstrate for the first time that during early infection, macrophages gained survival advantage owing to the antiapoptosis potency of SAG (Figures 1 and 2), which ubiquitinates proapoptosis factors, Bax and SARM (Figures 4 and 5 and Supplementary Figure S8). The SCF (Skp1-cullin-F-box proteins) is the largest family of E3 ubiquitin ligases, which is composed of four components: SKP1, a cullin, an F-box protein, and RBX1 or RBX2. The specificity of tetrameric SCF E3 ligase is determined by the F-box, which constitutes $\sim 70$ members. $^{23,24}$ To better understand cellular responses during infection, future work will be required to delineate the key components contributing to distinct SCFs in a modular manner.

Not just apoptosis ${ }^{25}$ but timely regulation of apoptosis is an important defense mechanism against pathogens. In our study, we observed minimal apoptosis of macrophages at early infection (Figure 2c), consistent with the notion that pathogens inhibit or delay the host's cell death for their own intracellular replication and survival. ${ }^{2,26}$ We provide 
a

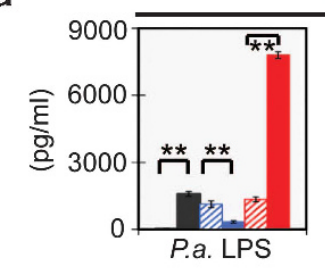

IL-1 $\beta$
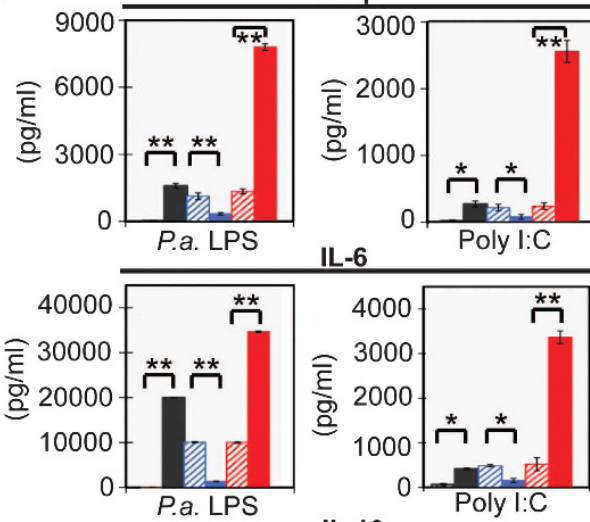

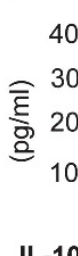
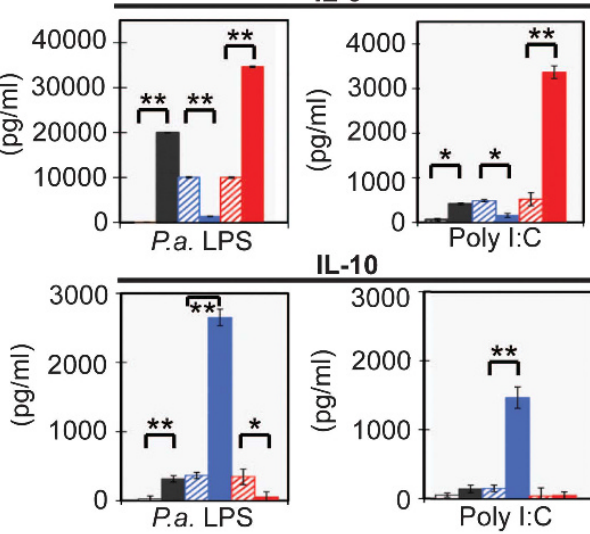

3000

产 $2000 \overbrace{0}^{3000}$

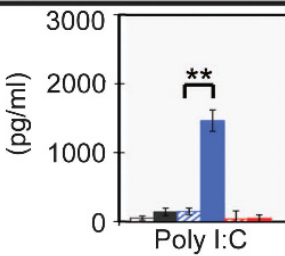

TNF- $\alpha$
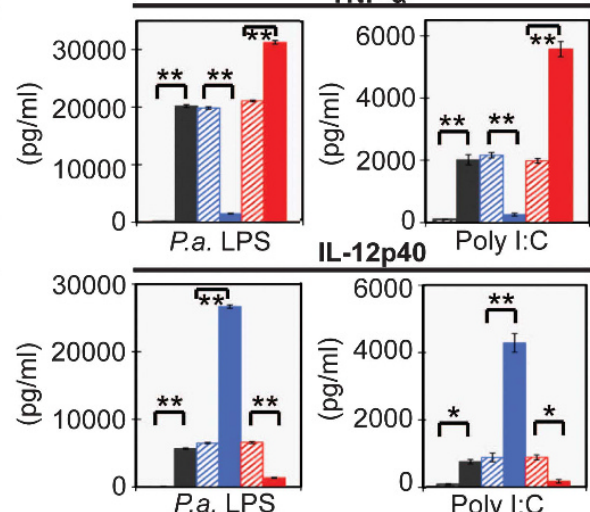

L-12p4

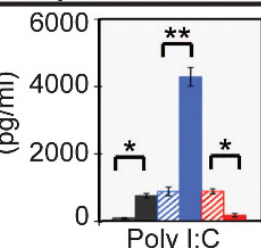

$\square$ PBS $\because$ Control siRNA+ PAMP

-PAMP $\square$ SAG SIRNA+ PAMP

$\because p c D N A+P A M P$

- HA-SAG-pcDNA + PAMP

b

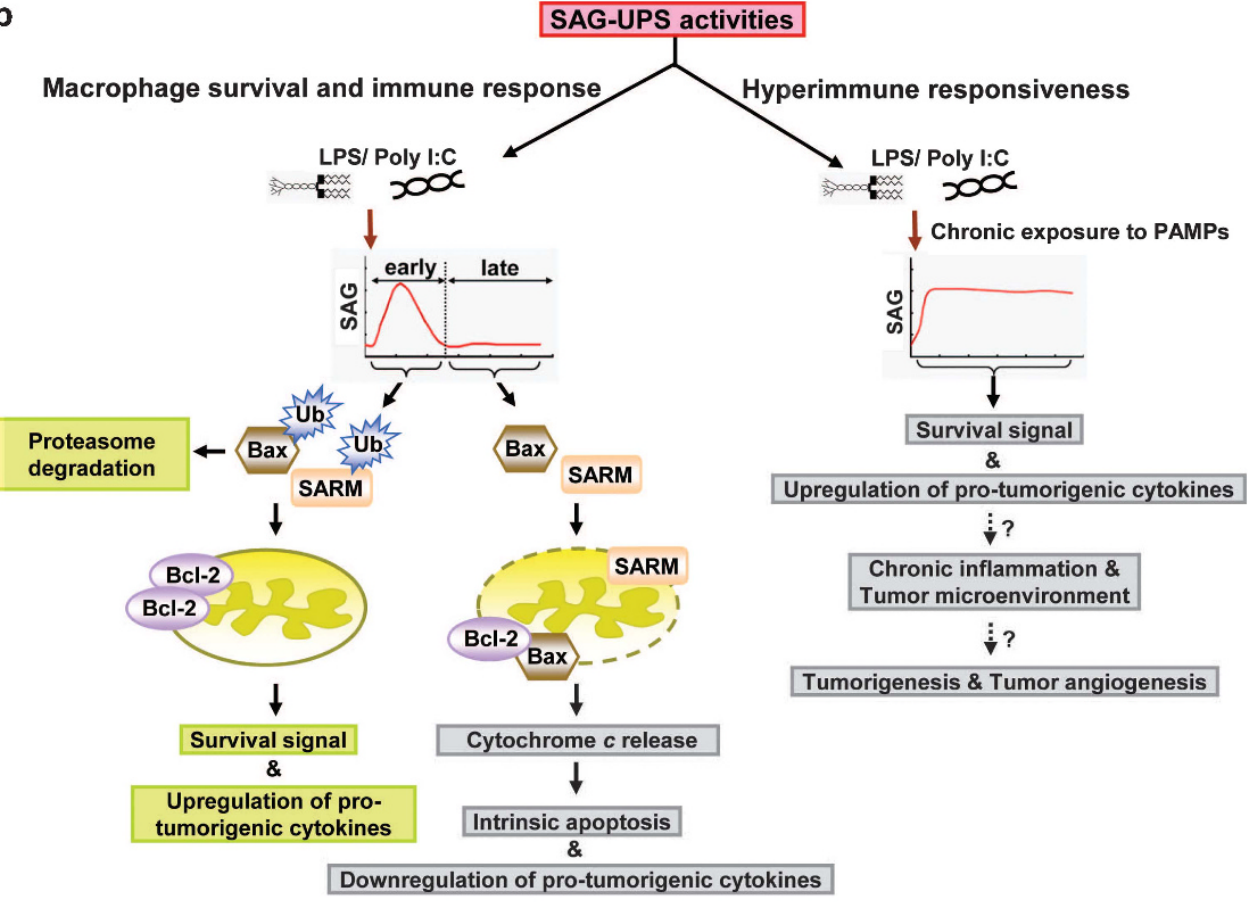

Figure 6 SAG-UPS upregulates protumorigenic cytokines, balances apoptosis and infection-inflammation in macrophages. (a) The levels of protumorigenic cytokines (IL-1 $\beta$, TNF- $\alpha$ and IL-6), antitumorigenic cytokine (IL-12p40) and anti-inflammatory cytokine (IL-10), in BMDM culture supernatants exposed to PAMP for $24 \mathrm{~h}$, were examined using enzyme-linked immunosorbent assay (ELISA) $(n=3)$. ${ }^{*} P<0.05 ;{ }^{*} P<0.01$. (b) A model showing a hypothetical mechanism of action of SAG-UPS during infection, which may lead to chronic inflammation and tumorigenesis. SAG-UPS maintains macrophage survival signal to facilitate immune response against infection at the early phase. SAG-mediated ubiquitination of cytosolic Bax and SARM is upregulated rapidly, thus sequestering these proapoptotic proteins away from the mitochondria. However, continuously overactivated SAG-UPS may induce hyperimmune responsiveness with uncontrolled survival signals in macrophages. Overactivated protumorigenic/ proangiogenic cytokines may create a tumor microenvironment, which favors proliferation of overactivated immune cells, causing them to switch into a tumorigenic mode. IL, interleukin; LPS, lipopolysaccharide; PBS, phosphate-buffered saline; poly I:C, polyinosinic-polycytidylic acid; siRNA, small interfering RNA; TNF, tumor necrosis factor; Ub, ubiquitin

compelling evidence for an apoptosis-escape mechanism of macrophages where apoptosis-related signaling pathways are inactivated via downregulation of proapoptotic proteins or upregulation of antiapoptotic proteins (Figures 2c and 3).
However, a significant increase in apoptosis at the later stage (>24 h; Figure 2c) indicates that persistent infection induces apoptosis, possibly to minimize the host's inflammatory response. ${ }^{27}$ Coincidentally, SARM, which regulates immune 
deactivation by its proapoptotic activity, ${ }^{9}$ was also found to respond dynamically to infection (Figure 3), albeit the reciprocal to that of SAG. We showed that SAG-UPS mediates ubiquitination and turnover of SARM (Figures 4 and 5 and Supplementary Figure S8), which probably ensures that an appropriate level of immune response is achieved before SARM-mediated apoptosis ensues. Thus, SAG-UPS controls SARM activity, which in turn regulates immune response and cell death on a periodic basis.

Bax is a key proapoptosis member of the $\mathrm{Bcl}-2$ family, which is regulated by UPS. ${ }^{28,29}$ In cancer cells, Bax was reported to undergo proteasomal degradation, although the involvement of SAG-mediated ubiquitination was untested. ${ }^{29}$ The turnover of Bax in immune cells and how it responds to an infection is hitherto unclear, although it is known that during stressinduced apoptosis, the imbalance between the mitochondrial $\mathrm{Bax}$ and Bcl-2 compromises mitochondrial integrity. ${ }^{30} \mathrm{We}$ found that consistent with the observation on SAG-mediated SARM turnover, Bax was susceptible to ubiquitin recognition and conjugation by SAG-UPS, thus reducing the half-life of the protein and disrupting the ratio of Bax and Bcl-2 (Figures 4 and 5 and Supplementary Figure S8). Furthermore, SAG opposes Bax translocation to the mitochondria (Figures $5 \mathrm{~d}$ and e), thus downregulating apoptosis. This mechanism explains the underlying resistance of macrophages to apoptosis during early infection.

To ascertain the downstream effects of SAG-UPS on infected macrophages, we examined the cytokine expression. In cancer growth SAG is overexpressed persistently, but in an infection, SAG accumulates to a peak at $12 \mathrm{~h}$ (Figures 1 and $5 \mathrm{a}$ and Supplementary Figure S1). Here, our observation of SAG-mediated upregulation of protumorigenic IL-6, TNF- $\alpha$ and IL-1 $\beta$ (Figure $6 \mathrm{a}$ ) is novel and indicates that SAG has a key role in inflammatory responses, facilitating macrophage survival during early infection. Supporting our finding are recent studies using MLN4924, an inhibitor of neddylation, which reduced the production of IL- 6 and TNF- $\alpha$ by dendritic cells. ${ }^{31}$ Figure $6 \mathrm{~b}$ (supported by experimental evidence of Figures 2-6a) depicts tightly controlled apoptosis of macrophages upon infection, showing that SAG-UPS is induced during early infection to counter apoptosis and to allow immune response. In contrast, SAG overexpression and SAG-mediated ubiquitination would continuously drive cell survival and infection-induced proinflammatory response (Figures 2 and $6 a$ ), which could conceivably create a chronic inflammatory state, attaining a tumor microenvironment. This may trigger immune overactivation and subsequently tumorigenesis. Recent studies using SAG deletion further highlighted the critical role of SAG in tumorigenesis ${ }^{32}$ and tumor angiogenesis. ${ }^{33}$ These interesting findings lend credence to our observation of the upregulation of protumorigenic cytokines (e.g. IL-6, TNF- $\alpha$ and IL-1 $\beta$ ), when immune cells undergo SAG overexpression. Corroboratively, these cytokines have also been referred to as proangiogenic cytokines. ${ }^{34,35}$ Recent evidence also suggests the correlation between persistent activation of TLRs at the sites of chronic inflammation and the development of cancer. ${ }^{36,37}$ Therefore, understanding how TLR signaling contributes to immune overactivation and the development of chronic inflammationassociated tumors may provide insights to link immunology and cancer biology. Future work using animal models with transient/conditional SAG knockdown may help to evaluate the impact of SAG responses on infection, disease progression and its potential contribution to the recruitment of immune cells to a tumor microenvironment under chronic infection.

In conclusion, we have demonstrated that macrophage life or death is synchronized by SAG-mediated ubiquitination of proapoptotic factors, which strategically sustains immune defense during early infection. Our findings clearly established the power of SAG-UPS as a functional link between immune defense and apoptosis or immune overactivation with a potential towards tumorigenesis. We propose SAG-UPS to be an efficient target for potential future development of immunomodulators.

\section{Materials and Methods}

Experiments were performed according to Institutional Animal Care and Use Committee guidelines (NUS protocol, 012/09).

Bacteria. $P$. aeruginosa (PAO-Iglewski) was grown in Luria-Bertani broth at $37^{\circ} \mathrm{C}$ to mid-log phase. After washing three times with PBS, the bacterial density and the colony-forming unit (CFU) were determined. The bacteria were heat inactivated at $56^{\circ} \mathrm{C}$ for $1 \mathrm{~h}^{38}$

Mouse J774, RAW264.7 cell lines, BMDM and reagents. Both J774 and RAW264.7 cells were maintained in DMEM supplemented with $10 \%$ fetal bovine serum. To verify the observations made with J774 and RAW264.7 cells, key experiments were performed with primary cells, using mouse BMDM. Briefly, bone marrow cells were isolated from the femur of 8- to 12-week-old BALB/c mice and incubated with $10 \mathrm{ng} / \mathrm{ml}$ mouse M-CSF (eBioscience, San Diego, CA, USA) for 7 days to allow differentiation. LPS from $P$. aeruginosa and $E$. coli (Sigma Chemical Co., St. Louis, MO, USA; L8643 and L4524, respectively) were used at $10 \mathrm{ng} / \mathrm{ml}$. Poly (I:C) and R848 (InvivoGen, San Diego, CA, USA) were used at $10 \mu \mathrm{g} / \mathrm{ml}$ and $25 \mathrm{ng} / \mathrm{ml}$, respectively. Proteasomes were effectively inhibited by $10 \mu \mathrm{M}$ MG132 (Sigma-Aldrich, St. Louis, MO, USA) for $6 \mathrm{~h}^{39}$ To evaluate the half-life of a protein, $80 \mu \mathrm{g} / \mathrm{ml} \mathrm{CHX}$ was administered. The primary antibodies used in this study were: SAG polyclonal antibody (Abcam, Cambridge, UK), Bcl-2 monoclonal antibody (Santa Cruz Biotechnology, Santa Cruz, CA, USA), Bax polyclonal antibody (Cell Signaling Technology, Inc., Danvers, MA, USA), SARM polyclonal antibody (Santa Cruz Biotechnology), Noxa polyclonal antibody (Santa Cruz Biotechnology), Bcl-xL polyclonal antibody (Cell Signaling Technology, Inc.), ubiquitin monoclonal antibody (Santa Cruz Biotechnology), GAPDH monoclonal antibody (Santa Cruz Biotechnology), VDAC polyclonal antibody (Cell Signaling Technology, Inc.), cytochrome $c$ monoclonal antibody (BD Biosciences, San Jose, CA, USA), control rabbit IgG (Santa Cruz Biotechnology), control mouse IgG (Santa Cruz Biotechnology) and a loading control, $\beta$-actin (Sigma Chemical Co.).

Real-time PCR. Total RNA was extracted with TRIzol reagent (Invitrogen) and the RNA was reverse transcribed using random hexamers and Superscript III (Invitrogen), according to the manufacturer's instructions. The synthesized cDNA was used for real-time PCR analysis using SYBR Green on a LightCycler 480 II instrument (Roche, Mannheim, Germany). The primers were SAG (109 bp product): sense, 5'-CAGGCTCCAAGTCGGGAGGCG-3', antisense, 5'-T GGACCCTGCAGATGGCACAGG-3'; Bcl-2 (350 bp product): sense, 5'-TACCGTC GTGACTTCGCAGAG- $3^{\prime}$, antisense, $5^{\prime}$-GGCAGGCTGAGCAGGGTCTT-3';0 Bax (160 bp product): sense, $5^{\prime}$-CGGCGAATTGGAGATGAACTG-3', antisense, $5^{\prime}$-GC AAAGTAGAAGAGGGCAACC- $3^{\prime} ;{ }^{40}$ SARM (132 bp product): sense, $5^{\prime}$-CATCACC CGCAAGAGGTTC-3', antisense, 5'-CCATAGGTGTACTGGCGGAA-3'; Noxa (130 bp product): sense, $5^{\prime}$-CCCAGATTGGGGACCTTAGT-3', antisense, $5^{\prime}$-CT GCGAACTCAGGTGGTAGC-3';1 Bcl-xL (399 bp product): sense, $5^{\prime}$-AGGCAGGCG ATGAGTTTGAAC- $3^{\prime}$, antisense, $5^{\prime}$-GAACCACACCAGCCACAGTCA-3 $3^{\prime} ;{ }^{40} \beta$-actin (103 bp product): sense, $5^{\prime}$-GCTGACAGGATGCAGAAGGAG-3', antisense, $5^{\prime}$-AG CCACCGATCCACACAGA-3'. All expression values were normalized based on $\beta$-actin as an endogenous control. 
SAG knockdown, HA-SAG overexpression and PAMP stimulation in J774, RAW264.7 macrophages or primary BMDM cells. SAG siRNA (Silencer select predesigned siRNA) and control (scrambled) siRNA were purchased from Ambion (Carlsbad, CA, USA) and Invitrogen, respectively. siRNA transfection into J774, RAW264.7 or BMDM cells (at $8 \times 10^{5}$ cells per well of a 6 -well plate) was conducted using $8 \mu \mathrm{l}$ X-tremeGENE Transfection Reagent (Roche) with 90 pmol of siRNAs per well. HA-SAG-pcDNA3 and pcDNA3 plasmids were used for SAG overexpression study. For BMDM cells, DNA transfection was conducted with $8 \times 10^{5}$ cells per well of a 6 -well plate using $4 \mu \mathrm{l}$ X-tremeGENE Transfection Reagent (Roche) with $2 \mu \mathrm{g}$ DNA plasmids. The transfection efficiency for siRNA knockdown was determined by immunodetection of SAG protein and real-time PCR of SAG mRNA (Supplementary Figure S2). The transfection efficiency for CDNA overexpression was determined by immunodetection of the HA-tag protein (Supplementary Figure S10). At $16 \mathrm{~h}$ after transfection, cells were treated with PAMP(s) for different time periods.

TMRE staining. Mitochondrial membrane potential was determined using TMRE (Sigma Chemical Co.), which stains intact mitochondria. Briefly, cells were loaded with $200 \mathrm{nM}$ TMRE dye for $20 \mathrm{~min}$ at $37^{\circ} \mathrm{C}$ to stain the mitochondrial matrix. The samples were then subjected to flow cytometric analysis for a minimum of 15000 events.

Caspase-9 and -3 assays. The J774 cells were transfected with SAGspecific siRNA or control scrambled siRNA for $16 \mathrm{~h}$, followed by PAMP stimulation at time points indicated. Apoptosis was confirmed by determining the caspase-specific cleavages of activated caspase- 9 and -3 (Oncolmmunin Inc., Gaithersburg, MD, USA). The fluorescence emission was measured at excitation $(552 \mathrm{~nm})$ and emission $(580 \mathrm{~nm})$ wavelengths, using a FACScan flow cytometer (Beckman Coulter, Inc., Brea, CA, USA).

Intracellular staining of representative apoptosis markers. The expression of SAG, Bcl-2, Bax and SARM was measured by staining intracellularly with specific antibodies. Briefly, cells were harvested at indicated time points and washed in PBS-TX (PBS containing $0.1 \%$ Triton X-100). The cells were incubated with primary antibody for 30 min, washed two times with PBS-TX and conjugated to secondary antibody, Alexa Fluor 488 (Invitrogen) or with phycoerythrin (Invitrogen) for $20 \mathrm{~min}$. After washing two times with PBS-TX, the cells were analyzed on a FACScan flow cytometer.

Cellular apoptosis assay. Early-stage apoptosis was measured using annexin V (eBioscience) in conjunction with 7-AAD (eBioscience). Annexin V identifies surface-exposed phosphatidylserine, and 7-AAD is retained in late apoptotic cells. Apoptosis was then analyzed using FACScan flow cytometer for a minimum of 20000 events.

Trypan Blue dye exclusion test. To assess total cell viability, cells were scraped and resuspended in equal volumes of culture medium and trypan blue dye $(0.05 \%$ solution; GIBCO-Invitrogen, Paisley, UK) and counted using a Neubauer improved hemocytometer.

MTT assay. To estimate cell death due to loss of mitochondrial activity at the end of PAMP treatments, the cells were incubated with $100 \mu \mathrm{l}$ MTT reagent (Sigma Chemical Co.) at $37^{\circ} \mathrm{C}$ for $3 \mathrm{~h}$. The metabolic activity of viable cells was measured at $570 \mathrm{~nm}$ using a BioTek microplate reader (Synergy Mx Monochromator, Winooski, VT, USA).

Subcellular fractionation, Co-IP and immunodetection. To prepare cytosolic and mitochondrial lysates, BMDM cells were homogenized and mitochondria were pelleted by a mitochondria isolation kit (Thermo Fisher Scientific, Rockford, IL, USA). The cytosolic fractions were subjected to Co-IP with anti-Bax or anti-SARM antibody. The mitochondrial fraction products were immunoblotted and probed sequentially with antibodies to Bax, SARM and VDAC. For Co-IP study with J774 or RAW264.7 total cell lysates, the cells were lysed in CHAPS lysis buffer containing $20 \mathrm{mM}$ Tris- $\mathrm{HCl}, \mathrm{pH} 7.5,5 \mathrm{mM} \mathrm{MgCl}$, $137 \mathrm{mM} \mathrm{KCl}$, $1 \mathrm{mM}$ EDTA, $1 \mathrm{mM}$ EGTA, $1 \%$ CHAPS and $1 \times$ protease inhibitors (complete EDTA-free cocktail, Roche). Then, the cell lysate was precleared by incubating with protein G Sepharose (GE Healthcare, Little Chalfont, UK) at $4{ }^{\circ} \mathrm{C}$ for $2 \mathrm{~h}$. The supernatant was incubated overnight at $4{ }^{\circ} \mathrm{C}$ with Bax or SARM or $\mathrm{Bcl}-2$ antibody, followed by a 3 -h incubation with protein $\mathrm{G}$ Sepharose. The washed immunoprecipitates, resuspended in Laemmli buffer, was boiled at $95{ }^{\circ} \mathrm{C}$ for 5 min before immunodetection of SAG, Bax, SARM, Bcl-2 or ubiquitin.

ELISA. To determine the effects of SAG on cytokine release, BMDM cells were transiently transfected with SAG-specific siRNA, control siRNA, HA-SAG or empty vector for $16 \mathrm{~h}$, followed by PAMP stimulation for $24 \mathrm{~h}$. The levels of IL-6, TNF- $\alpha$, $\mathrm{IL}-1 \beta$, IL-12p40 and IL-10, secreted by the cells, were quantified in 24-h supernatants by using OptEIA Mouse ELISA Set (BD Biosciences).

Statistical analysis. Data are presented as means \pm S.D. of three independent experiments, with three replicates per sample/condition tested. Differences between averages were analyzed by two-tailed Student's $t$-test. Significance was set at $P$-value of $<0.05\left({ }^{\star} P<0.05,{ }^{* *} P<0.01\right)$. All target signals from western blots were quantified by Scion Image software (Frederick, MD, USA). The acquired data from FACS were analyzed with Summit software (Version 4.3.02, Fort Wayne, IN, USA).

\section{Conflict of Interest}

The authors declare no conflict of interest.

Acknowledgements. We thank Dr. BH Iglewski (University of Rochester) for P. aeruginosa strain PAO-Iglewski; Dr. Yi Sun (University of Michigan, USA) for plasmids HA-SAG-pcDNA3 and pcDNA3, and Drs Zhu Yong and Laishram Pradeepkumar Singh for proofreading the manuscript. This work was supported by grants from the A*STAR Biomedical Research Council, Singapore (10/1/21/19/658) and the National Medical Research Council, Singapore (NMRC/CBRG/0055/2014).

1. Subramanian K, Du R, Tan NS, Ho B, Ding JL. CD163 and IgG codefend against cytotoxic hemoglobin via autocrine and paracrine mechanisms. J Immunol 2013; 190: $5267-5278$

2. Rudel T, Kepp O, Kozjak-Pavlovic V. Interactions between bacterial pathogens and mitochondrial cell death pathways. Nat Rev Microbiol 2010; 8: 693-705.

3. Hasnain SE, Begum R, Ramaiah KV, Sahdev S, Shajil EM, Taneja TK et al. Host-pathogen interactions during apoptosis. J Biosci 2003; 28: 349-358.

4. Neutzner A, Li S, Xu S, Karbowski M. The ubiquitin/proteasome system-dependent control of mitochondrial steps in apoptosis. Semin Cell Dev Biol 2012; 23: 499-508.

5. Ding JL, Tan KC, Thangamani S, Kusuma N, Seow WK, Bui THH et al. Spatial and temporal coordination of expression of immune response genes during Pseudomonas infection of horseshoe crab, Carcinoscorpius rotundicauda. Genes Immun 2005; 6: 557-574.

6. Panneerselvam P, Singh LP, Ho B, Chen J, Ding JL. Targeting of pro-apoptotic TLR adaptor SARM to mitochondria: definition of the critical region and residues in the signal sequence. Biochem J 2012; 442: 263-271.

7. Mink M, Fogelgren B, Olszewski K, Maroy P, Csiszar K. A novel human gene (SARM) at chromosome $17 \mathrm{q} 11$ encodes a protein with a SAM motif and structural similarity to Armadillo//beta-catenin that is conserved in mouse, Drosophila, and Caenorhabditis elegans. Genomics 2001; 74: 234-244.

8. Carty M, Goodbody R, Schroder M, Stack J, Moynagh PN, Bowie AG. The human adaptor SARM negatively regulates adaptor protein TRIF-dependent Toll-like receptor signaling. Nat Immunol 2006; 7: 1074-1081.

9. Panneerselvam P, Singh LP, Selvarajan V, Chng WJ, Ng SB, Tan NS et al. T-cell death following immune activation is mediated by mitochondria-localized SARM. Cell Death Differ 2013: 20: 478-489

10. Duan H, Wang Y, Aviram M, Swaroop M, Loo JA, Bian J et al. SAG, a novel zinc RING finger protein that protects cells from apoptosis induced by redox agents. Mol Cell Biol 1999: 19: 3145-3155

11. Jia L, Yang J, Hao X, Zheng M, He H, Xiong X et al. Validation of SAG/RBX2/ROC2 E3 ubiquitin ligase as an anticancer and radiosensitizing target. Clin Cancer Res 2010; 16: 814-824

12. Kondratieva TK, Kobets NV, Khaidukov SV, Yeremeev VV, Lyadova IV, Apt AS et al. Characterization of $T$ cell clones derived from lymph nodes and lungs of Pseudomonas aeruginosa-susceptible and resistant mice following immunization with heat-killed bacteria. Clin Exp Immunol 2000; 121: 275-282

13. Allan SM, Rothwell NJ. Cytokines and acute neurodegeneration. Nat Rev Neurosci 2001; 2: 734-744.

14. Bingle L, Brown NJ, Lewis CE. The role of tumour-associated macrophages in tumour progression: implications for new anticancer therapies. J Pathol 2002; 196: 254-265.

15. Etoh T, Shibuta K, Barnard GF, Kitano S, Mori M. Angiogenin expression in human colorectal cancer: the role of focal macrophage infiltration. Clin Cancer Res 2000; 6 : 3545-3551. 
16. Pinto A, Morello S, Sorrentino R. Lung cancer and Toll-like receptors. Cancer Immunol Immunother 2011; 60: 1211-1220.

17. Berlato C, Cassatella MA, Kinjyo I, Gatto L, Yoshimura A, Bazzoni F. Involvement of suppressor of cytokine signaling-3 as a mediator of the inhibitory effects of IL-10 on lipopolysaccharide-induced macrophage activation. J Immunol 2002; 168: 6404-6411.

18. Park P-h, McMullen MR, Huang H, Thakur V, Nagy LE. Short-term treatment of RAW264.7 macrophages with adiponectin increases tumor necrosis factor- $\alpha$ (TNF- $\alpha$ ) expression via ERK1/2 activation and Egr-1 expression: role of TNF- $\alpha$ in adiponectin-stimulated interleukin-10 production. J Biol Chem 2007; 282: 21695-21703.

19. Wang L, Zhao Y, Qian J, Sun L, Lu Y, Li H et al. Toll-like receptor-4 signaling in mantle cell lymphoma. Cancer 2013; 119: 782-791.

20. Gu Q, Tan M, Sun Y. SAG/ROC2/Rbx2 is a novel activator protein-1 target that promotes c-Jun degradation and inhibits 12-0-tetradecanoylphorbol-13-acetate-induced neoplastic transformation. Cancer Res 2007; 67: 3616-3625.

21. Tan M, Zhao Y, Kim S-J, Liu M, Jia L, Saunders T et al. SAG/RBX2/ROC2 E3 ubiquitin ligase is essential for vascular and neural development by targeting NF1 for degradation. Dev Cell 2011; 21: 1062-1076.

22. Wei D, Sun Y. Small RING finger proteins RBX1 and RBX2 of SCF E3 ubiquitin ligases: the role in cancer and as cancer targets. Genes Cancer 2010; 1: 700-707.

23. Cenciarelli C, Chiaur DS, Guardavaccaro D, Parks W, Vidal M, Pagano M. Identification of a family of human F-box proteins. Curr Biol 1999; 9: 1177-S1173.

24. Winston JT, Koepp DM, Zhu C, Elledge SJ, Harper JW. A family of mammalian F-box proteins. Curr Biol 1999; 9: 1180-S1183.

25. Lamkanfi M, Dixit VM. Manipulation of host cell death pathways during microbial infections. Cell Host Microbe 2010; 8: 44-54.

26. Brune W. Inhibition of programmed cell death by cytomegaloviruses. Virus Res $2011 ; 157$ 144-150.

27. Behar SM, Divangahi M, Remold HG. Evasion of innate immunity by Mycobacterium tuberculosis: is death an exit strategy? Nat Rev Microbiol 2010; 8: 668-674.

28. Liu F-T, Agrawal SG, Gribben JG, Ye H, Du M-Q, Newland AC et al. Bortezomib blocks Bax degradation in malignant $B$ cells during treatment with TRAIL. Blood 2008; 111: 2797-2805.

29. Li B, Dou QP. Bax degradation by the ubiquitin/proteasome-dependent pathway: involvement in tumor survival and progression. Proc Natl Acad Sci 2000; 97: 3850-3855.

30. Youle RJ, Strasser A. The BCL-2 protein family: opposing activities that mediate cell death Nat Rev Mol Cell Biol 2008; 9: 47-59.

31. Mathewson N, Toubai T, Kapeles S, Sun Y, Oravecz-Wilson K, Tamaki H et al. Neddylation plays an important role in the regulation of murine and human dendritic cell function. Blood 2013; 122: 2062-2073.
32. Li H, Tan M, Jia L, Wei D, Zhao Y, Chen $G$ et al. Inactivation of SAG/RBX2 E3 ubiquitin ligase suppresses $\mathrm{Kras}^{\mathrm{G} 12 \mathrm{D}}$-driven lung tumorigenesis. J Clin Invest 2014; 124: 835-846.

33. Tan M, Li H, Sun Y. Endothelial deletion of Sag/Rbx2/Roc2 E3 ubiquitin ligase causes embryonic lethality and blocks tumor angiogenesis. Oncogene 2013; e-pub ahead of print 11 November 2013; doi:10.1038/onc.2013.473.

34. Fan Y, Ye J, Shen F, Zhu Y, Yeghiazarians Y, Zhu W et al. Interleukin-6 stimulates circulating blood-derived endothelial progenitor cell angiogenesis in vitro. J Cereb Blood Flow Metab 2007; 28: 90-98.

35. Naldini A, Carraro F. Role of inflammatory mediators in angiogenesis. Curr Drug Targets Inflamm Allergy 2005; 4: 3-8.

36. Rakoff-Nahoum S, Medzhitov R. Toll-like receptors and cancer. Nat Rev Cancer 2009; 9: 57-63.

37. Chen R, Alvero AB, Silasi DA, Steffensen KD, Mor G. Cancers take their Toll-the function and regulation of Toll-like receptors in cancer cells. Oncogene 2008; 27: 225-233.

38. Tertilt C, Joh J, Krause A, Chou P, Schneeweiss K, Crystal RG et al. Expression of B-cell activating factor enhances protective immunity of a vaccine against Pseudomonas aeruginosa. Infect Immun 2009; 77: 3044-3055.

39. Wu H-M, Wen H-C, Lin W-W. Proteasome inhibitors stimulate interleukin-8 expression via ras and apoptosis signal-regulating kinase-dependent extracellular signal-related kinase and c-Jun N-terminal kinase activation. Am J Resp Cell Mol Biol 2002; 27 234-243.

40. Exley GE, Tang C, McElhinny AS, Warner CM. Expression of caspase and BCL-2 apoptotic family members in mouse preimplantation embryos. Biol Reprod 1999; 61: 231-239.

41. Knowlton JJ, Dermody TS, Holm GH. Apoptosis induced by mammalian reovirus is beta interferon (IFN) independent and enhanced by IFN regulatory factor $3-$ and NF- $\mathrm{\kappa B}$ dependent expression of Noxa. J Virol 2012; 86: 1650-1660.

(i) (-) $\Theta$ This work is licensed under a Creative Commons Attribution-NonCommercial-NoDerivs 3.0 Unported License. The images or other third party material in this article are included in the article's Creative Commons license, unless indicated otherwise in the credit line; if the material is not included under the Creative Commons license, users will need to obtain permission from the license holder to reproduce the material. To view a copy of this license, visit http://creativecommons.org/licenses/by-nc-nd/3.0/

Supplementary Information accompanies this paper on Cell Death and Differentiation website (http://www.nature.com/cdd) 